\title{
Avaliação do método Penman-Piche para a estimativa da evapotranspiração de referência em U beraba, MG $^{1}$
}

\author{
André L. T. Fernandes ${ }^{2}$, Eusímio F. Fraga Júnior ${ }^{3} \&$ Bruno Y. Takay $^{4}$
}

\begin{abstract}
RESU MO
O bjetivou-se, no presente trabalho, realizar a comparação do método de determinação da evapotranspiração de referência, Penman-M onteith com o método de Penman-Piché, que utiliza medidas de evaporação pelo evaporímetro de piche. Analisou-se o período de janeiro de 2003 a dezembro de 2008, trabalhando-se com dados diários de temperatura do ar, radiação solar, umidade relativa e velocidade do vento, coletados em uma estação meteorológica automatizada, localizada na Fazenda Experimental da U niube, M G. Para a comparação dos métodos de estimativa de evapotranspiração de referência foram utilizados: coeficientes de correlação (r), índice de concordância de Willmott (d) e 0 índice de confiança (c), que é o produto entre " $r$ " e " $d$ ". Concluiu-se, no trabalho, que é possível estimar a evapotranspiração de referência de maneira satisfatória através da evaporação medida pelo evaporímetro de piche, quando se obtiveram coeficientes de correlação acima de 0,90 . N os meses de baixa umidade relativa do ar a ETo estimada pelo método que considera as medidas do evaporímetro de piche, apresenta redução na precisão, com valores de " $r$ " de 0,70; 0,78 e 0,70, respectivamente para os meses de junho, agosto e setembro da série estudada.
\end{abstract}

Palavras-chave: evaporímetro, irrigação, atmômetro

\section{Evaluation of Penman-Piche method to estimate reference evapotranspiration in U beraba, MG}

\begin{abstract}
The present research aimed to accomplish a comparison between the reference method of estimation of evapotranspiration, Penman-M onteith, considered as the standard method by FAO, with Penman-Piché method, which uses measurements of the evaporation from the Piché's evaporimeter. The period from January 2003 to December 2008 was analyzed considering daily data of air temperature, solar radiation, relative humidity of air and wind speed, which were collected with an automatic weather station, located in the Experimental Farm of U niversity of U beraba, in U beraba, in the State of M inas Gerais, Brazil. Correlation coefficient ( $r$ ), Willmott agreement index (d) and confidence index (c), which is the product between " $r$ " and " $d$ ", were used in the comparison between reference evapotranspiration estimated by Penman-M onteith (ETpm) and Penman-Piche (ETpi). It was concluded that it is possible to estimate the reference evapotranspiration in a satisfactory manner through the evaporation measured by the Piche's evaporimeter. In the months of low relative humidity of air, the evapotranspiration estimated by the method which considers the measures of Piche's evaporimeter shows reduction in precision, with " $r$ " values of 0,$70 ; 0,78$ and 0,70 , respectively for the months of June, August and September of the studied series.
\end{abstract}

Key words: evaporimeter, irrigation, atmometer

\footnotetext{
Trabalho financiado, em parte, pelo Consórcio Brasileiro de Pesquisa e Desenvolvimento do Café - CBP\&D/Café

2 Universidade de U beraba, Av. N enê Sabino 1801, Bloco M, CEP 38055-500, U beraba, M G. Fone: (34) 3319-8963, Fax: (34) 3314-8910. E-mail: andre.fernandes@uniube.br

${ }^{3}$ M estrando ESALQ/USP, Av. Pádua Dias 11, CEP 13418-900, Piracicaba, SP. Fone: (19) 3447-8579, ramal 24. E-mail: eusimio@ymail.com ${ }^{4}$ Engenheiro Agrônomo, Syngenta Proteção de Cultivos, Rua Presidente Vargas 1280. Edifício das Palmeiras, Sala 804, Centro, CEP 38340-000, U berlândia, MG . Fone: (34) 9912-5612. E-mail: bruno@takay.com.br
} 


\section{INTRODUÇÃO}

A determinação da quantidade de água necessária para as culturas é um dos principais parâmetros para o planejamento correto, dimensionamento e manejo de qualquer sistema de irrigação. Sua quantificação é realizada fazendo-se o balanço hídrico da camada do solo ocupada pelo sistema radicular da cultura, o qual tem, na "evapotranspiração" e na precipitação pluviométrica, seus principais componentes (Papaioannou et al., 1996; Volpe, 2003). A evapotranspiração compreende simultaneamente a evaporação e a transpiração (Righi et al., 2002), processos controlados pelo suprimento de água às plantas e pela disponibilidade de energia resultante da interação com as variáveis meteorológicas que condicionam a demanda atmosférica (Pivetta et al., 2010). Ocorre naturalmente como forma de dissipar energia e manter o metabolismo para o crescimento e desenvolvimento do vegetal. O termo evapotranspiração de referência (ETo) foi definido como perdas por evaporação e transpiração vegetal que ocorrem em uma extensa superfície coberta com grama de 0,08 a $0,15 \mathrm{~m}$, em crescimento ativo, cobrindo totalmente o solo e sem deficiência de água.

Segundo Sousa et al. (2010), a ETo é uma variável relevante para o planejamento de irrigação, por ser afetada apenas pelos fatores climáticos. Em geral, os métodos de estimativa da ETo são baseados em variáveis climáticas, muitos deles na temperatura, radiação ou métodos combinados (radiação e temperatura).

Apesar da existência de diversos modelos para se estimar a ETo, eles são utilizados em condições climáticas e agronômicas muito diferentes daquelas em que, inicialmente, foram concebidos e, por isto, é de extrema importância avaliar o grau de exatidão desses modelos antes de utilizá-los para nova condição (Silva et al., 2005; Buriol, 2001; Sousa et al., 2010). A não-verificação da adequação dos métodos de estimativa da evapotranspiração de referência às condições climáticas, a falta de precisão na estimativa e o erro, devido ao uso de instrumentos de medidas inadequados em geral, também conduzem ao manejo inadequado da água, acarretando em aplicações insuficientes ou em excesso resultando em perdas e prejuízos consideráveis às plantas e ao solo diminuindo, desta forma, a eficiência do uso de irrigação.

Um procedimento mais eficaz estaria em conduzir o processo de estimativa da evapotranspiração em uma só etapa, descartando-se a utilização dos coeficientes de cultura. Para isto, sugere-se que o método de Penman-Monteith seja adotado como padrão, com a utilização de valores adequados de resistência aerodinâmica e de resistência do dossel, específicas para cada cultura. Entretanto, a adoção imediata desse método incorreria em algumas dificuldades na obtenção de valores confiáveis para a resistência do dossel (Heldwein et al., 2004; Leitão et al., 2007).

Pereira et al. (2002) citam vários fatores que podem interferir na evapotranspiração das culturas, entre eles: a abertura dos estômatos, a reflectância, a rugosidade aerodinâmica, a extensão da área coberta pelo vegetal, a estação do ano, a disponibilidade de energia, a demanda atmosférica e o suprimento de água do solo às plantas. Outros fatores que podem influenciar nas taxas de evapotranspiração são as condições do solo, os fertilizantes, as infestações de pragas e doenças, as práticas agrícolas e a irrigação (Blanco \& Folegatti, 2004). A altitude também afeta diretamente as temperaturas do solo e do ar e a pressão atmosférica, que são fatores passíveis de influenciar a evapotranspiração. Para dado local a disponibilidade de radiação é controlada pelo poder refletor da superfície, que é expresso pelo coeficiente de reflexão (albedo); superfícies mais claras refletem mais que as escuras e, portanto, têm menos energia disponível; a demanda atmosférica é controlada pelo poder evaporante do ar e, quanto mais seco estiver o ar, maior será a demanda atmosférica. No entanto, existe inter-relação entre a demanda pelo ar e o suprimento de água pelo solo (Pereira et al., 2002).

$\mathrm{Na}$ escolha de um método para determinação da evapotranspiração, devem ser levadas em consideração praticidade e precisão (Back, 2007; Dalmago et al., 2001). Apesar de esses métodos teóricos e micrometereológicos serem baseados em princípios físicos, apresentam limitações, principalmente quanto à instrumentação, o que pode restringir a utilização. Dentro deste contexto, o presente trabalho teve por objetivo comparar o método de determinação da evapotranspiração de referência padrão, Penman-Monteith (ETpm), com o método de estimativa utilizando o evaporímetro de Piche (ETpi), no denominado Método de Penman-Piché, propondo equações de regressão para cada período do ano. $\mathrm{O}$ evaporímetro de Piche, que faz medida diária do poder evaporante do ar à sombra, tem custo de aquisição muito baixo em relação a outros equipamentos meteorológicos e, dependendo das condições ambientais, pode estimar satisfatoriamente a evapotranspiração, facilitando o manejo da irrigação.

\section{MATERIAL E MÉTODOS}

O experimento foi implantado e conduzido em Uberaba, região do Triângulo Mineiro do Estado de Minas Gerais, cuja latitude é de $19^{\circ} 44^{\prime} 13^{\prime \prime} \mathrm{S}$, longitude de $47^{\circ} 57^{\prime} 27^{\prime \prime} \mathrm{W}$ e altitude de $850 \mathrm{~m}$, na Fazenda Experimental da Universidade de Uberaba. A área experimental estava localizada no centro de uma área de $20 \mathrm{~m}^{2}$, cultivada com grama, circundada por uma área de 22,6 ha de café. O clima de Uberaba, segundo a classificação de Köeppen, é do tipo Aw, tropical quente úmido, com inverno frio $\left(15 / 16^{\circ} \mathrm{C}\right)$ e seco. As médias anuais de precipitação e temperatura são de $1.474 \mathrm{~mm}$ e $22,6^{\circ} \mathrm{C}$, respectivamente. As condições climáticas se enquadram na classificação de irrigação suplementar/complementar.

Os dados diários de temperatura do ar, radiação solar, umidade relativa e velocidade do vento foram coletados a partir de uma estação agrometeorológica automática, da marca METOS, modelo Micrometos 300, que possibilitou a estimativa da evapotranspiração pelo Método de Penman-Monteith, conforme descrito e parametrizado no Boletim da FAO n.56 (Allen et al., 1998). A estação meteorológica automática tem dimensões reduzidas $(27,0 \mathrm{~cm}$ de comprimento $\mathrm{x} 11,5 \mathrm{~cm}$ de diâmetro) no centro da estrutura. A estação consiste de um coletor de dados com $512 \mathrm{~Kb}$ de memória não volátil (PCB), um 
monitor de cristal líquido (LCD) para mostrar os valores registrados, uma porta de infravermelho para comunicação com computador pessoal, um protetor de radiação direta para impedir os sensores de temperatura e umidade relativa de ficarem superaquecidos com a luz do sol incidindo diretamente, e uma conexão de entrada com os seguintes sensores: a) Temperatura e umidade relativa do ar: foram medidos com uma unidade que combina um sensor SME 160-30 para temperatura e um sensor HC 200 para umidade relativa. O sensor de temperatura tem uma faixa de operação de $-30 \mathrm{a}+90^{\circ} \mathrm{C}$, com precisão de $0,5^{\circ} \mathrm{C}$. O sensor de umidade relativa apresenta uma faixa de operação de 10 a $98 \%$ de umidade relativa, com precisão de 3 a 4\%; b) Insolação: montado diretamente no $\mathrm{PCB}$, um fotorresistor foi usado como sensor. O limiar de duração do dia é ajustado para aproximadamente 300 lux, sendo a faixa de medida de 0 a 2000 lux; c) Radiação solar global: utilizou-se um sensor do tipo fotocélula especialmente projetada para absorver a luz numa faixa de 400 nanômetros (nm) a $1000 \mathrm{~nm}$ de comprimento de onda. A faixa de medida está entre 0 a $2000 \mathrm{~W} \mathrm{~m}^{-2}$; d) Velocidade de vento: $\mathrm{O}$ anemômetro utilizado opera num intervalo de valores entre $0,1 \mathrm{~m} \mathrm{~s}^{-1}$ e $40 \mathrm{~m} \mathrm{~s}^{-1}$. As variáveis monitoradas foram registradas automaticamente a partir do sensor infravermelho, que estabelecia o contato entre a estação e um microcomputador portátil.

A medida diária do poder evaporante do ar à sombra foi realizada com o auxílio de um evaporímetro de Piche (ETpi) instalado dentro de um abrigo, a 1,5 m, situado na mesma área em que a estação agrometeorológica se encontrava. Este equipamento consiste de um tubo de vidro calibrado e fechado numa das extremidades, com comprimento de $350 \mathrm{~mm}$; um diâmetro externo de $15 \mathrm{~mm}$; escala em 300 divisões que corresponde a décimo de milímetro de altura de água evaporada; o diâmetro do disco de papel tem aproximadamente $30 \mathrm{~mm}$. O tubo é cheio de água destilada, fechado com um papel de filtro circular e preso com uma mola. O papel umedecido evapora, baixando o nível de água dentro do tubo. Medidas consecutivas permitem calcular a evaporação no período desejado, um dia no caso específico deste trabalho. O método de Penman-Monteith é também chamado combinado, pois associa os efeitos de balanço de energia com o poder evaporante do ar. Stanhill (1962), nas condições áridas de Israel, utilizou a evaporação medida pelo evaporímetro de Piche $(\mathrm{Pi}$, $\mathrm{mm} \mathrm{d}^{-1}$ ) no abrigo meteorológico, para estimar o termo aerodinâmico da equação de Penman, ou seja, Eq. 1:

$$
(1-w) \times E=0,1468 \times P i+0,118
$$

em que:

Pi - evaporação obtida pelo evaporímetro de Piché, $\mathrm{mm} \mathrm{d}^{-1}$

w - definido por Makkink (1957) como sendo uma função da temperatura do ar (Eq. 2 e 3):

$$
\begin{gathered}
\mathrm{W}=0,407+0,0145 \times \mathrm{T}<\mathrm{T}<16{ }^{\circ} \mathrm{C} \\
\mathrm{w}=0,483+0,01 \times \mathrm{T}<\mathrm{T}<32{ }^{\circ} \mathrm{C}
\end{gathered}
$$

Esta aproximação, segundo Pereira et al. (2002), tem a vantagem de dispensar medidas de velocidade do vento e o déficit de saturação, visto que o evaporímetro de Piche é um indicador do poder evaporante do ar. Usando valores médios mensais da evapotranspiração potencial em gramado e valores médios de evaporação de tanque de $20 \mathrm{~m}^{2}$, Villa-Nova \& Ometto (1981), encontraram que (Eq. 4):

$$
\mathrm{ETPi}=\frac{(0,28 \times \mathrm{Pi})}{(1-\mathrm{w})}
$$

Com este trabalho, procurou-se comparar as estimativas de evapotranspiração pelo método de Penman-Piché (ETpi) com os valores estimados pelo Método de Penman Monteith (ETpm), padrão FAO, no período de 2003 a 2008. Este método é físico, baseado no método original de Penman, introduzindo os conceitos de resistência do dossel $\left(r_{c}\right)$ e de resistência aerodinâmica $\left(\mathrm{r}_{\mathrm{a}}\right)$ (Monteith, 1965). O método de Penman Monteith considera que a ET é proveniente dos termos energético e aerodinâmico, os quais são controlados pelas resistências ao transporte de vapor da superfície para a atmosfera. O cálculo foi feito pela seguinte expressão (Eq. 5):

$$
\begin{aligned}
& \mathrm{ETpm}=\frac{0,408 \times \mathrm{s} \times(\mathrm{Rn}-\mathrm{G})+\frac{\gamma \times 900 \times \mathrm{U}_{2} \times\left(\mathrm{e}_{\mathrm{s}}-\mathrm{e}_{\mathrm{a}}\right)}{\mathrm{T}+273}}{\mathrm{~s} \times \gamma \times\left(1+0,34 \times \mathrm{U}_{2}\right)} \\
& s=\frac{(4098 \mathrm{es})}{(237,3+\mathrm{T})^{2}} \\
& \mathrm{es}=\frac{\left(\mathrm{es}^{\mathrm{T} \max }+\mathrm{es}^{\mathrm{T} \min }\right)}{2} \\
& \text { es } \left.^{T}=0,611 \times 10^{[(7,5 \times T) /(237,3+T)}\right] \\
& \mathrm{ea}=\frac{\left(\mathrm{UR}_{\text {med }} \times \mathrm{es}\right)}{100} \\
& \mathrm{UR}_{\text {med }}=\frac{\left(\mathrm{UR}_{\text {max }}+\mathrm{UR}_{\text {min }}\right)}{2} \\
& \mathrm{~T}=\frac{\left(\mathrm{T}_{\max }+\mathrm{T}_{\min }\right)}{2}
\end{aligned}
$$

em que:

$\mathrm{R}_{\mathrm{n}}$ - radiação solar líquida total diária, $\mathrm{mm} \mathrm{d}^{-1}$

$\mathrm{G}^{\mathrm{n}}$ - fluxo de calor no solo, $\mathrm{MJ} \mathrm{m}^{-2} \mathrm{~d}^{-1}$

$\mathrm{g}$ - constante psicrométrica $=0,063 \mathrm{kPa}{ }^{\circ} \mathrm{C}^{-1}$

$\mathrm{T}$ - temperatura média do $\mathrm{ar},{ }^{\circ} \mathrm{C}$

$\mathrm{U}_{2}$ - velocidade do vento a $2 \mathrm{~m}$ de altura, $\mathrm{m} \mathrm{s}^{-1}$

$\mathrm{e}_{\mathrm{a}}$ - pressão de saturação do vapor d'água, $\mathrm{kPa}$

$\mathrm{e}_{\mathrm{s}}$ - pressão de saturação do vapor d’água, $\mathrm{kPa}$ 
$\mathrm{S} \quad$ - declividade da curva de pressão de vapor na temperatura do ar, $\mathrm{kPa}^{\circ} \mathrm{C}^{-1}$

$\mathrm{T}_{\max }$ - temperatura máxima do ar, ${ }^{\circ} \mathrm{C}$

$\mathrm{T}_{\min }$ - temperatura mínima do ar, ${ }^{\circ} \mathrm{C}$

$\mathrm{UR}_{\max }$ - umidade relativa máxima, $\%$

$\mathrm{UR}_{\text {min }}$ - umidade relativa mínima, \%

Além deste aspecto, buscou-se também, identificar uma equação que se ajustasse melhor ao modelo de PenmanMonteith-FAO e fosse de utilização mais simples, como é o caso das equações que dependem apenas de temperatura do ar, evaporação e insolação. Para a comparação, inicialmente os dados foram analisados por mês, individualmente, em seguida, a média dos meses, nos anos avaliados e, por último, para as estações do ano. Para a análise dos dados foram utilizados: coeficiente de correlação (r), índice de concordância de Willmott (Willmott, 1981) (d) e o índice de confiança (c), que é o produto entre r e d, proposto por Camargo \& Sentelhas (1997). Na Tabela 1 são apresentados os valores do índice "c" e sua classificação e, na Tabela 2, a classificação para "r", seguindo-se a metodologia de Hopkins (2007). Finalmente, com base nos

Tabela 1. Classificação do índice de confiança (c)

\begin{tabular}{cc}
\hline Coeficiente de confiança (c) & Desempenho \\
$>0,85$ & Ótimo \\
0,76 a 0,85 & Muito Bom \\
0,66 a 0,75 & Bom \\
0,61 a 0,65 & Mediano \\
0,51 a 0,60 & Sofrível \\
0,41 a 0,50 & Mau \\
$<0,40$ & Péssimo \\
\hline Fonte: Camargo \& Sentelhas (1997) &
\end{tabular}

Tabela 2. Classificação das correlações de acordo com o coeficiente de correlação $(r)$

\begin{tabular}{cc}
\hline Coeficiente de correlação $(\mathbf{r})$ & Desempenho \\
$0,0-0,1$ & Muito baixa \\
$0,1-0,3$ & Baixa \\
$0,3-0,5$ & Moderada \\
$0,5-0,7$ & Alta \\
$0,7-0,9$ & Muito Alta \\
$0,9-1,0$ & Quase perfeita \\
\hline
\end{tabular}

dados obtidos pelo método do ETpi, propôs-se um ajuste visando à melhoria da precisão e, sobretudo, da exatidão desses métodos em relação à equação de Penman-Monteith-FAO, para a condição deste estudo.

\section{RESULTADOS E DISCUSSÃO}

Na Tabela 3 são apresentados os dados de evapotranspiração obtidos por ambos os métodos, durante o período analisado. A evapotranspiração calculada pelo método de Penman Monteith foi superior à evapotranspiração estimada pelo método de Penman Piché nos anos de 2003, 2004, 2007 e 2008, respectivamente em 205, 109, 56 e 40 mm anuais. Nos anos de 2005 e 2006 houve superestimativa do método de Penman Piché de 120 e $121 \mathrm{~mm}$, respectivamente, para os anos de 2005 e 2006.

$\mathrm{Na}$ Tabela 4 constam os valores de correlação, exatidão e confiança dos dados para os dois métodos de estimativa de evapotranspiração (coeficiente de correlação " $r$ ") para a região de Uberaba, nos períodos de 2003 a 2008. Ressalta-se que, para o mês de janeiro, ocorreu a melhor correlação entre métodos, com $r$ = 0,96; já no mês de junho se obteve a correlação mais baixa durante o ano, com $r=0,695$. Analisando-se o índice de confiança (índice c), os melhores resultados também foram obtidos nos meses

Tabela 4. Valores dos coeficientes de correlação " $r$ ", de exatidão "d" e de confiança " $c$ " e da classificação do desempenho dos diferentes métodos usados para a estimativa da evapotranspiração de referência (ETo)

\begin{tabular}{lcccc} 
Meses & $\mathbf{R}^{\mathbf{2}}$ & $\mathbf{d}$ & $\mathbf{C}$ & Desempenho \\
J aneiro & 0,95 & 0,95 & 0,91 & Ótimo \\
Fevereiro & 0,84 & 0,90 & 0,75 & Bom \\
Março & 0,95 & 0,96 & 0,91 & Ótimo \\
Abril & 0,90 & 0,94 & 0,85 & Ótimo \\
Maio & 0,91 & 0,94 & 0,86 & Ótimo \\
J unho & 0,70 & 0,77 & 0,54 & Sofrível \\
Julho & 0,90 & 0,81 & 0,73 & Bom \\
Agosto & 0,78 & 0,85 & 0,66 & Bom \\
Setembro & 0,70 & 0,73 & 0,51 & Sofrível \\
Outubro & 0,93 & 0,96 & 0,89 & Ótimo \\
Novembro & 0,95 & 0,97 & 0,92 & Ótimo \\
Dezembro & 0,93 & 0,94 & 0,88 & Ótimo \\
\hline
\end{tabular}

Tabela 3. Valores médios mensais da ETo, em mm d-1, avaliados no período de 2003 a 2008

\begin{tabular}{|c|c|c|c|c|c|c|c|c|c|c|c|c|}
\hline \multirow{3}{*}{ Meses } & \multicolumn{12}{|c|}{ ETo - PM (Penman-Monteith) / Epi (Piche) } \\
\hline & \multicolumn{2}{|c|}{2003} & \multicolumn{2}{|c|}{2004} & \multicolumn{2}{|c|}{2005} & \multicolumn{2}{|c|}{2006} & \multicolumn{2}{|c|}{2007} & \multicolumn{2}{|c|}{2008} \\
\hline & ETpm & ETpi & ETpm & ETpi & ETpm & ETpi & ETpm & ETpi & ETpm & ETpi & ETpm & ETpi \\
\hline Janeiro & 3,1 & 3,0 & 3,2 & 3,3 & 2,8 & 3,4 & 2,5 & 3,0 & 2,7 & 3,2 & 3,0 & 3,2 \\
\hline Fevereiro & 4,1 & 3,4 & 3,8 & 3,3 & $* *$ & $* *$ & 3,1 & 3,6 & 4,1 & 4,4 & 3,1 & 3,2 \\
\hline Março & 3,1 & 2,9 & 4,5 & 4,3 & 3,1 & 3,6 & 3,1 & 3,6 & 4,3 & 4,6 & 3,3 & 3,6 \\
\hline Abril & 2,6 & 2,4 & 3,6 & 3,5 & 3,5 & 4,0 & 3,3 & 3,7 & 3,7 & 4,0 & 3,1 & 3,2 \\
\hline Maio & 3,7 & 3,2 & 3,0 & 3,0 & 3,0 & 3,4 & 3,3 & 3,6 & 3,3 & 3,5 & 3,0 & 3,2 \\
\hline J unho & 3,9 & 3,3 & 3,3 & 3,2 & 2,9 & 3,3 & $* *$ & $* *$ & 3,6 & 4,0 & 3,1 & 3,2 \\
\hline Julho & 4,5 & 3,4 & 3,8 & 3,4 & 3,1 & 3,4 & 3,7 & 3,9 & 3,3 & 3,3 & 3,9 & 3,7 \\
\hline Agosto & 4,5 & 3,6 & 5,3 & 4,3 & 4,1 & 4,4 & 4,2 & 4,3 & 5,0 & 4,8 & 4,2 & 4,2 \\
\hline Setembro & 5,3 & 4,2 & 6,4 & 5,3 & 3,4 & 3,5 & 3,9 & 4,0 & 5,6 & 2,1 & 4,6 & 1,9 \\
\hline Outubro & 4,4 & 3,7 & 4,6 & 4,2 & 4,3 & 4,3 & 3,1 & 3,5 & 4,9 & 4,7 & 3,9 & 4,0 \\
\hline Novembro & 3,9 & 3,7 & 4,1 & 4,0 & 3,4 & 3,9 & 3,3 & 3,7 & 3,7 & 3,5 & 3,5 & 3,8 \\
\hline Dezembro & 4,4 & 4,1 & 3,5 & 3,8 & 2,9 & 3,4 & 3,3 & 3,8 & 3,6 & 3,9 & 3,6 & 3,7 \\
\hline
\end{tabular}

** Não houve coleta de dados devido à manutenção dos instrumentos de coleta 
de maior precipitação, com destaque para janeiro, março, abril, maio, outubro, novembro e dezembro, com valores de "c", respectivamente, de 0,$91 ; 0,91 ; 0,85 ; 0,86 ; 0,89 ; 0,92$ e 0,88 .

Ferreira (1972), avaliando métodos de estimativa da ETo para Campinas, Pindamonhangaba e Ribeirão Preto, estado de São Paulo, observou que os valores médios da ETo obtidos pelo evaporímetro de Piche apresentaram ajustes satisfatórios em relação ao método de Penman Monteith, comportamentos esses semelhantes aos obtidos neste trabalho.

Observou-se que as melhores correlações foram obtidas nos meses de janeiro, março, abril, maio, outubro, novembro e dezembro, com coeficientes de correlação (r) acima de 0,90. Pôde-se observar, também, que o coeficiente de exatidão ficou acima de 0,90, exceto para os meses de junho, julho, agosto e setembro, meses que apresentaram o "d"entre 0,73 e 0,85 . Os mesmos meses que apresentaram baixo índice de exatidão também indicaram a mais baixa classificação de confiança.

Na Figura 1 são apresentadas as relações entre a ETo estimada pelos dois métodos em cada uma das estações do ano; tal formatação permite uma análise mais detalhada sobre as variações da evaporação de Piche (ETpi) ao longo do ano. Pelas altas correlações obtidas $(r>0,73)$ nas estações do ano, recomenda-se o uso das equações propostas por estação específica (Figura 1) para a estimativa da evapotranspiração

A.

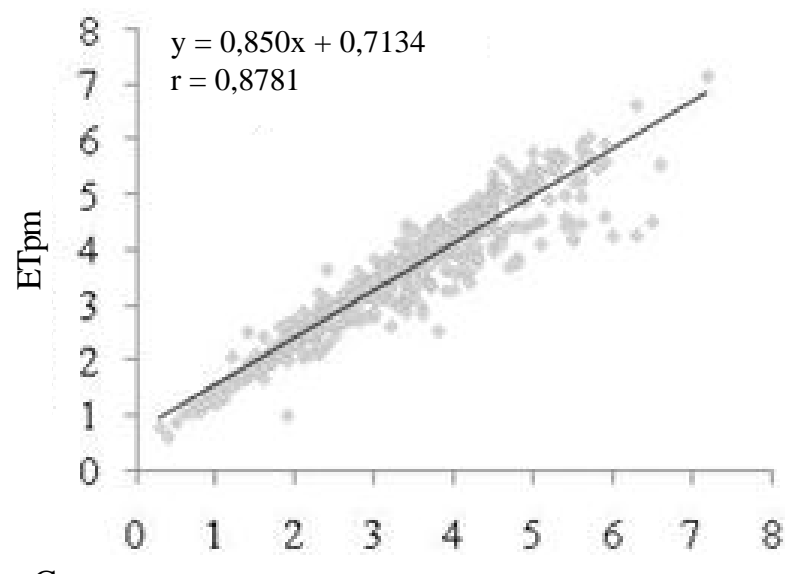

C.

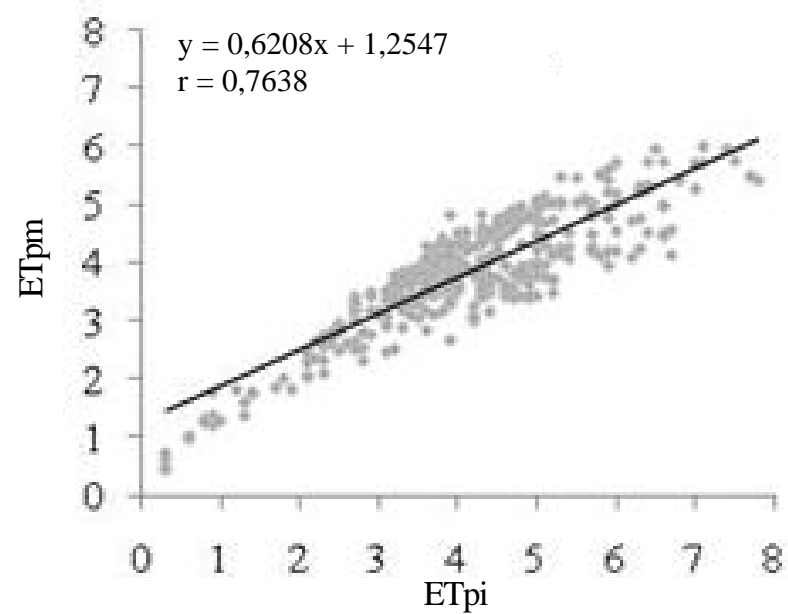

de referência, pela facilidade que este procedimento apresenta, ou seja, emprega apenas os dados medidos no evaporímetro de Piche.

Em ambiente protegido, Heldwein et al. (2001) obtiveram, ao correlacionar a evapotranspiração direta da cultura do pimentão por unidade de índice de área foliar (ETmf) com a obtida com o evaporímetro de Piche (ETpi), um ajuste significativo $(\mathrm{r}=0,78)$, considerando-o satisfatório, e um bom instrumento para a determinação da ETo de culturas em estufa plástica e, consequentemente, para ser empregado no manejo hídrico das mesmas.

Observou-se queda de precisão nas estimativas, na medida em que se iniciou a estação de inverno. Conclusões semelhantes foram apresentadas por Duarte et al. (2003), que observaram que as condições ambientais contribuem para a diferenciação da evapotranspiração durante as estações do ano, fato que se deve aos valores de umidade relativa do ar, radiação disponível, temperatura ambiente e à intensidade dos ventos. Por outro lado, no evaporímetro de Piche (ETpi), que se localiza dentro de um abrigo, tais fatores são insignificantes, pois o que passa a comandar a evaporação de forma mais intensa, é a capacidade (maior ou menor) do ar ambiente em reter vapor d'água, sendo que a variável determinante seria a umidade relativa do ar, fato confirmado conforme a Figura 2.

B.
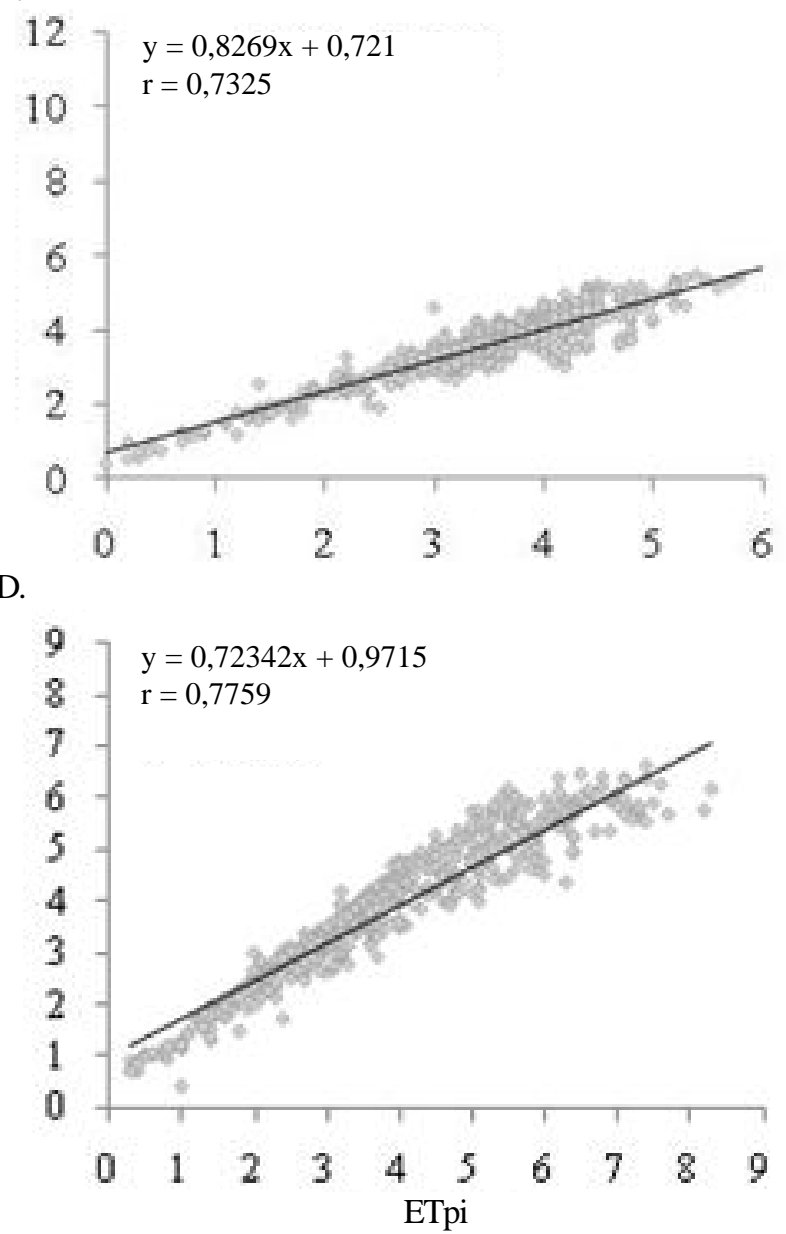

Figura 1. Relação entre a ETo estimada $\left(\mathrm{mm}_{\mathrm{di}} \mathrm{a}^{-1}\right)$ pelos métodos de Penman M onteith (ETpm) e de Penman-Piche (ETpi), para a região de U beraba, MG ; $(A)$ verão; (B) outono; $(C)$ inverno e (D) primavera 


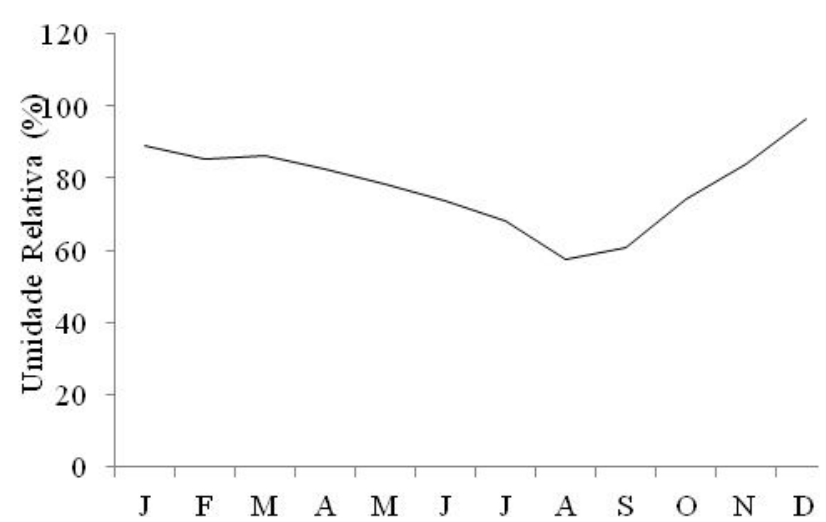

Figura 2. Variação média mensal da umidade relativa do ar (\%) ao longo do ano, no período de 2003 a 2008, na Fazenda Escola da U niversidade de U beraba, U beraba, MG

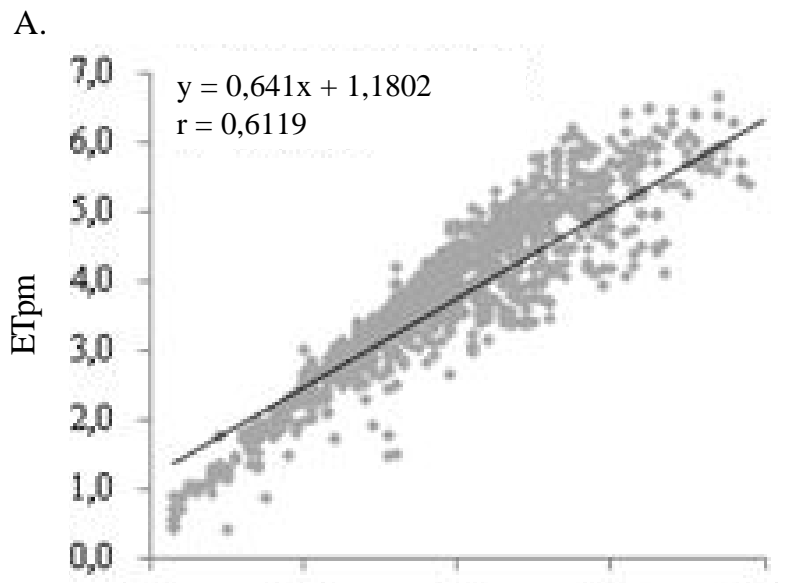

B.

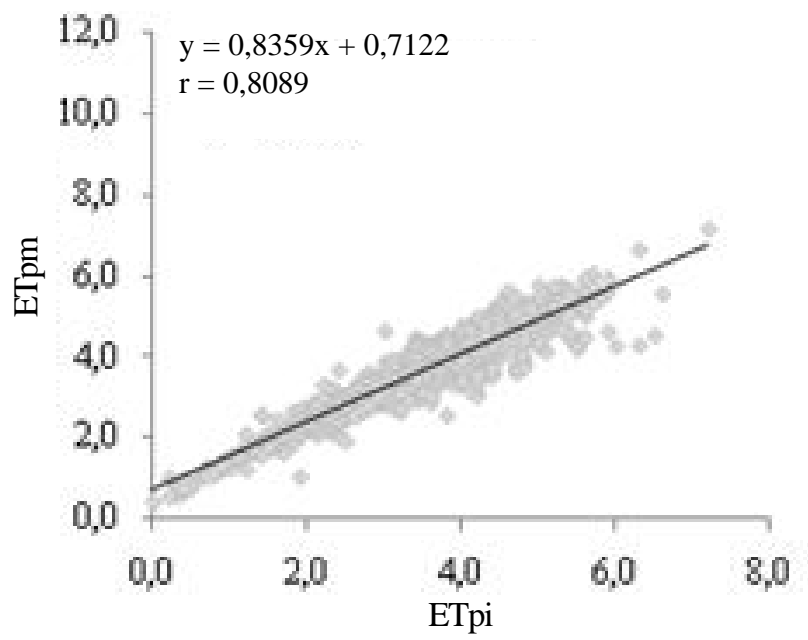

Figura 3. Relação entre a ETo ( $\left.\mathrm{mm} \mathrm{dia}^{-1}\right)$ estimada pelos métodos de $P M$ e de Penman-Piche, para a região de U beraba, M G; $(A)$ verão-outono; $(B)$ inverno - primavera

Ao se agruparem os dados em dois grupos: estação VerãoOutono e estação Inverno - Primavera verificou-se baixa correlação no período Inverno-Primavera, período este influenciado pela ação da umidade relativa do ar (UR\%), conforme demonstrado na Figura 2. Vescove \& Turco (2005), comparando o método de estimativa de ETo por PenmanMonteith e pelo método do Tanque Classe A, obtiveram correlações semelhantes ao agruparem as estações do ano, sendo que, em suas medições, o coeficiente de determinação $\left(\mathrm{R}^{2}\right)$ para verão-outono foi de $0,83 \mathrm{e}$, para inverno-primavera, de 0,62 (Figura 3 ).

\section{CONCLUSÕES}

1. É possível estimar a evapotranspiração de referência de maneira satisfatória através da evaporação medida pelo evaporímetro de piché.

2. As estimativas de evapotranspiração com o evaporímetro de piche foram menos precisas e exatas apenas em dois meses do ano, junho e setembro.

3. O evaporímetro de piché, devido ao seu baixo custo e facilidade de operação, pode ser alternativa viável para programas de manejo da irrigação.

\section{LITERATURA CITADA}

Allen R. G.; Pereira, L. S.; Raes, D.; Smith, M. Crop evapotranspiration. Rome: FAO, 1998. 297p. Irrigation and Drainage Paper, 56.

Back, A. J. Variação da evapotranspiração de referência calculada em diferentes intervalos de tempo. Engenharia Agrícola, v.27, p.139-145, 2007.

Blanco, F. F.; Folegatti, M. V. Evaluation of evaporationmeasuring equipments for estimating evapotranspiration within a greenhouse. Revista Brasileira de Engenharia Agrícola e Ambiental. v.8, p.184-188, 2004.

Buriol, G. A. Evaporação d'água em estufas plásticas e sua relação com o ambiente externo: 2 - avaliação do uso do tanque classe A e do evaporímetro de Piche. Revista Brasileira de Agrometeorologia, v.9, p.35-41, 2001.

Camargo, A. P.; Sentelhas, P. C. Avaliação do desempenho de diferentes métodos de estimativa da evapotranspiração potencial no Estado de São Paulo. Revista Brasileira de Agrometeorologia, v.5, p.89-97, 1997.

Dalmago, G. A.; Heldwein, A. B.; Buriol, G. A.; Wilsmann, S.; Trentin, G.; Tazzo, I. F. Avaliação de métodos para determinação da evapotranspiração máxima da cultura do pimentão em estufa plástica. Revista Brasileira de Agrometeorologia, v.9, p.201-211, 2001.

Duarte, W. O.; Barros, D. L.; Assunção, W. L. Comparação entre as leituras diárias do tanque classe "A" e o evaporímetro de piche, da estação climatológica da UFU. In: Simpósio Regional de Geografia Perspectivas para o Cerrado no Século XXI, 2, 2003, Uberlândia. Anais... Uberlândia: UFU, 2003, p.6-7.

Ferreira, W. A. Utilização da evaporação "piche" na estimativa da evapotranspiração potencial. Botucatu: UNESP, 1972. 130p. Tese Doutorado

Heldwein, A. B.; Dalmago, G. A.; Streck, L.; Tazzo, I. F.; Trentin, G. Utilização do evaporímetro de Piche exposto à radiação solar para estimar a evapotranspiração máxima do pimentão em estufa plástica. Revista Brasileira de Agrometeorologia, v.9, p.213-217, 2001. 
Heldwein, A. B. ; Streck, L. ; Schneider, F. M.; Grimm, E. L.; Nied, A. H.; Tazzo, I. F. Modelos para estimativa da evapotranspiração máxima da abóbora italiana em estufa plástica. Revista Brasileira de Agrometeorologia, v.12, p.7586, 2004.

Hopkins,W. G. Correlation coefficient. <http://www.sportsci.org/ resource/stats/correl.html>. 04 Nov. 2007.

Leitão, M. de M. V. B. R.; Oliveira, G. M. de; Leitão, T. J. V. Avaliação do desempenho de diferentes métodos de estimativa da evaporação para duas regiões da Paraíba. Revista Brasileira de Engenharia Agrícola e Ambiental, v.11, p.585-593, 2007.

Makkink, G. F. Ekzameno de la formula de Penman. Netherlands Journal of Agricultural Science, v.5, p.290-305, 1957.

Monteith, J. L. Evaporation and environment. Symposia of the Society for Experimental Biology, v.19, p.205-234, 1965.

Papaioannou, G.; Vouraki, K.; Kerkides. P. Piche evaporimeter data as a substitute for Penman equation's aerodynamic term. Agricultural and Forest Meteorology, v.82, p.83-92, 1996.

Pereira, A. R.; Angelocci, L. R.; Sentelhas, P. C. Agrometeorologia: Fundamentos e aplicações práticas. Guaíba: Agropecuária, 2002.478p.

Pivetta, C. R.; Heldwein, A. B.; Maldaner, I. C.; Radons, S. R.; Tazzo, I. F.; Lucas, D. D. Evapotranspiração máxima do pimentão cultivado em estufa plástica em função de variáveis fenométricas e meteorológicas. Revista Brasileira de Engenharia Agrícola e Ambiental, v.14, p.768-775, 2010.

Righi, E. Z.; Angelocci, L. R.; Buriol, G. A.; Heldwein, A. B. Transpiração do tomateiro cultivado em estufa plástica e suas relações com a radiação solar e déficit de saturação do ar. Revista Brasileira de Agrometeorologia, v.10, p.9-18, 2002.
Silva, V. de P. R. da; Belo Filho, A. F.; Silva, B. B. da; Campos, J. H. B. da C. Desenvolvimento de um sistema de estimativa da evapotranspiração de referência. Revista Brasileira de Engenharia Agrícola e Ambiental, v.9, p.547$553,2005$.

Sousa, I. F. de; Silva, V. P. R. da; Sabino, F. G.; Netto, A. de O.; Silva, B. K. N.; Azevedo, P. V. Evapotranspiração de referência nos perímetros irrigados do estado de Sergipe. Revista Brasileira de Engenharia Agrícola e Ambiental, v.14, p.633-644, 2010.

Stanhill, G. The use of the Piche evaporimeter in the calculation of evaporation. Quarterly Journal of the Royal Meteorological Society, v.88, p.80-82, 1962.

Streck, L. Evapotranspiração máxima e coeficiente de cultura da abóbora italiana em estufa plástica. Revista Brasileira de Agrometeorologia, v.11, p.43-52, 2003.

Vescove, H. V.; Turco, J. E. P. Comparação de três métodos de estimativa da evapotranspiração de referência para a região de Araraquara - SP. Engenharia Agrícola, v.25, p.713-721, 2005.

Villa-Nova, N. A.; Ometto, J. C. Adaptação e simplificação do método de Penman às condições climáticas do Estado de São Paulo. In: Simpósio Brasileiro de Hidrologia e Recursos Hídricos, 4, 1981, Fortaleza. Anais... Fortaleza: SBRH, 1981. p.281-299.

Volpe, C. A.; Oliveira, A. D. de, Relações entre a evaporação medida em diferentes evaporímetros. Revista Brasileira de Agrometeorologia, v.11, p.245-252, 2003.

Willmott, C. J. On the validation of models. Physical Geography, v.2, p.184-194, 1981. 